\title{
El confinamiento por COVID-19 podría aumentar el riesgo de síndrome de fragilidad en adultos mayores
}

\section{Confinement for COVID-19 could increase the risk of frailty in elderly}

$\mathrm{DOI}$

https://doi.org/10.35434/rcmhnaaa.2021.14Sup1.1192

Señor editor:

No es de nuevo conocimiento la situación sanitaria global actual por la pandemia de COVID-19 y el cómo esta, vino a cambiar el modo de vida de toda la población, aún a estas fechas. Como Ballena bien lo resalta en el artículo "Impacto del confinamiento por COVID-19 en la calidad de vida y salud mental", publicado en su revista, el confinamiento fue y es en algunas regiones obligatorio en los adultos mayores por ser el grupo más vulnerable ante esta enfermedad ${ }^{(1)}$. En el contexto de la pandemia, el que los familiares o cuidadores tuvieran que alejarse de los adultos mayores aumenta el riesgo de accidentes dentro de casa como caídas, así mismo predispone a un sentimiento de soledad que puede conllevar a la aparición de síndromes geriátricos, entre ellos síndrome de fragilidad.

El aislamiento social es un estado objetivo de tener poco contacto social, mientras que la soledad es un sentimiento subjetivo ${ }^{(2)}$, que aunque no siempre van juntos, uno puede ser reflejo del otro. Se estima que el aislamiento aumenta en la población de adultos mayores en un 50\% la aparición de demencia, en un $29 \%$ la incidencia de enfermedades coronarias y en $32 \%$ la de eventos cerebrovasculares ${ }^{(2)}$.

La fragilidad es resultado de múltiples factores de entre ellos destacan la pobreza, vivir solo, bajo nivel educativo, la polifarmacia, enfermedades concomitantes, depresión, mal nutrición y baja actividad física ${ }^{(3)}$, este último es magnificado por el confinamiento puesto que al no tener compañía y el tener que estar aislado se ve reflejado en sedentarismo ${ }^{(1)}$, no solo en jóvenes y niños.

Existen estudios que muestran una coexistencia entre depresión y síndrome de fragilidad en hasta el $53,8 \%$ de los pacientes geriátricos ${ }^{(4)}$. Entender la fragilidad como un estado de decremento en la reserva fisiológica que conlleva a una vulnerabilidad ${ }^{(4)}$, permite entender la relevancia de tomar medidas contra el sentimiento de soledad para evitar desenlaces adversos en la salud de los adultos mayores. El estudio realizado en México por Herrera-Badilla revelan una asociación entre soledad y fragilidad con un OR de 2,7 con un IC $95 \%$ de $1,08-6,98$ y un valor de $p$ de $0,03^{(5)}$.

Ballena y sus colaboradores afirman que la salud mental de la población general efectivamente se vio comprometida por el confinamiento debido a la pandemia ${ }^{(1)}$, pero es necesario hacer un énfasis importante en los adultos mayores, puesto que son población vulnerable no solo a graves desenlaces por COVID-19, si no a los que indirectamente genera el aislamiento social sobre ellos, los cuales los ponen en riesgo de hospitalización e incluso en complicaciones serias como la muerte ${ }^{(3)}$.

Por lo anterior expuesto, la población de adultos mayores debe ser vigilada y atendida en sus necesidades, evitando en lo posible el sentimiento de soledad que puede
R. Abraham Del Val-Gómez ${ }^{1,2, a}$

FILIACIÓN

1. Centro Médico de Especialidades, Ciudad Juárez, Chihuahua, México.

2. Universidad Autónoma de Ciudad Juárez, Ciudad Juárez, Chihuahua, México.

a. Médico Interno de Pregrado.

ORCID

1. R. Abraham Del Val-Gómez 0000-0002-0094-3549

CORRESPONDENCIA

R. Abraham Del Val-Gómez

Teléfono: 526561326175

EMAIL

ra.delval@hotmail.com

CONFLICTOS DE INTERÉS

Los autores niegan conflictos de interés.

FINANCIAMIENTO

Autofinanciamiento.

REVISIÓN DE PARES

Recibido: 06/09/2021

Aceptado: 20/09/2021

COMO CITAR

Del Val-Gómez, A. El confinamiento por COVID19 podría aumentar el riesgo de síndrome de fragilidad en adultos mayores. Revista Del Cuerpo Médico Hospital Nacional Almanzor Aguinaga Asenjo, 2021, 14(Sup1), 105 - 106. https://doi.org/10.35434/rcmhnaaa.2021.14S up1.1192 
encaminar a la aparición de fragilidad y con ello llegar a desenlaces desfavorables.

Sería interesante y relevante valorar la asociación entre síndromes geriátricos y el confinamiento por la pandemia de COVID-19. Agradezco su lectura.

\section{REFERENCIAS BIBLIOGRÁFICAS}

1. Ballena CL, Cabrejos L, Dávila Y, Gonzales CG, Mejía GE, Ramos V, et al. Impacto del confinamiento por COVID-19 en la calidad de vida y salud mental. Rev del Cuerpo Médico Hosp Nac Almanzor Aguinaga A s e n jo. $2021 ; 14(1): 87$ - 9 . https://cmhnaaa.org.pe/ojs/index.php/rcmhnaaa/article/view/90 4/411
2. Wu B. Social isolation and loneliness among older adults in the context of COVID-19: a global challenge. Glob Heal Res Policy.

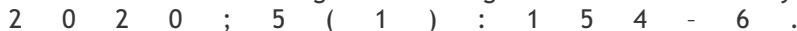
https://ghrp.biomedcentral.com/articles/10.1186/s41256-02000154-3

3. Dent E, Kowal $P$, Hoogendijk EO. Frailty measurement in research and clinical practice: A review. Eur J Intern Med [Internet]. 2016;31:3-10. Available from: http://dx.doi.org/10.1016/j.ejim.2016.03.007

4. Vaughan L, Corbin AL, Goveas JS. Depression and frailty in later life: A systematic review. Clin Interv Aging. 2015;10:1947-58. https://www.ncbi.nlm.nih.gov/pmc/articles/PMC4687619/

5. Herrera-Badilla A, Navarrete-Reyes AP, Amieva H, Avila-Funes JA Loneliness is associated with frailty in community-dwelling elderly adultsJags march 2011-vol. 59, no. 3 letters to the editor. JAGS.

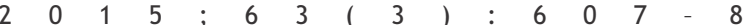
https://agsjournals.onlinelibrary.wiley.com/doi/10.1111/jgs.13308 